\title{
Der «Stich» von 1564 - eine primäre Lungenpest
}

Von Vera Waldis

Der «Stich» von 1564 - so 1848 bezeichnet von dem Medizinhistoriker Konrad Meyer-Ahrens (1813-1872) ${ }^{1}$ - erweist sich nach unserem heutigen medizinischen Wissen nicht als «typhöse» Krankheit, sondern als primäre Lungenpest. Es geht hier aber weniger darum, eine retrospektive Diagnose zu stellen, als vielmehr, die Diagnose des Johannes Weyer (1515-1588) ${ }^{2}$, eines genau beobachtenden und selbständigen Arztes, zu erhärten.

Heute kennen wir zwei Pestformen: die Lungen- und die Bubonen- oder Beulenpest. Beide werden von dem gleichen Erreger, der Yersinia pestis, hervorgerufen; sie unterscheiden sich aber durch ihre Übertragungsart. Die Bubonenpest entsteht durch den Biß eines infizierten Flohs, wobei sich der Erreger zuerst in der Lymphbahn und in die Lymphdrüsen ausbreitet. Von hier kann er in die Blutbahn gelangen. Die primäre Lungenpest wird von Mensch zu Mensch übertragen, nachdem der Erreger von der Blutbahn aus die Lungen sekundär befällt, in die Ausatmungsluft gerät und bei einer Kontaktperson die primäre Pestpneumonie auslöst. Seit den 1950er Jahren ist auch eine sogenannte primäre nicht-pneumonische Lungenpest bekannt. Mit diesem Ausdruck meint der Pestforscher Robert Pollitzer die neuerdings oft diagnostizierten Fälle von Pest-Anginen, deren Verlauf manchmal schwer, manchmal aber so milde ist, daß die Krankheitsträger sich völlig gesund fühlen. Ähnlich wie von den Pestbeulen kann der Erreger von den Tonsillen aus in die Blutbahn einbrechen oder mit der Einatmungsluft schließlich doch in die Lungen gelangen ${ }^{3}$.

Die mittelalterlichen Autoren des Schwarzen Todes, insbesondere 1348 Guy de Chauliac (ca. 1300-1368) ${ }^{4}$, betrachteten beide Formen - Lungenwie Bubonenpest - als ein und dieselbe Krankheit. In der Neuzeit aber, bis zum Ende des 19. Jahrhunderts, geriet diese Betrachtungsweise in Vergessenheit. Die meisten Ärzte sahen in den Bubonen und Anthrazes (Blasen im Bereiche des Flohbisses) die einzigen Kriterien, welche die Diagnose «Pest» zuließen. Andere epidemische, schnell tödlich verlaufende Krankheiten und Krankheitsmodalitäten wurden unter die «pestilenzischen Fieber» gerechnet. Noch Georg Sticker mißt in seinem Monumentalwerk über die Pest (1908-10) der primären Lungenpest nur geringe Bedeutung zu, und im historischen Teil erwähnt er sie überhaupt nicht. 
Johannes Weyer beschrieb zwei ihm unbekannte, neue Seuchen in seinen «Medicae Observationes Rarae» unter dem Titel: Über die verschiedenen Arten von pestilenzischen Flüssen, die 1564 und 1565 an gewissen Orten Niederdeutschlands auftraten ${ }^{5}$. Weyer berichtet, daß 1564 eine weitverbreitete, heftige Pest auftrat; sie ließ sich in Konstantinopel, in Alexandria wie in Lyon, London, Danzig, Augsburg, Wien, Köln und am ganzen Oberrhein bis Basel nachweisen. Dagegen sei sie am Niederrhein viel milder gewesen. Doch hatte Weyer hier mit Halsschwellungen und gefährlichen, rasch tödlich verlaufenden Anginen zu kämpfen, welche sowohl in ihrer Kontagiosität als auch Letalität der Bösartigkeit einer Pest gleichkamen. Manche der Erkrankten starben schon nach einem, andere nach drei oder vier Tagen.

Die Krankheit manifestierte sich zuerst mit Fieber und Erbrechen. Später trat eine Schwellung im Bereiche der Zunge auf, die sowohl die Kehle als auch die Speiseröhre verschloß, so daß einige Kranke daran erstickten. Manchmal konnte man keine Geschwulst im Halsbereich wahrnehmen, dafür aber eine «Phrenitis», das Krankheitsbild einer Hirnentzündung. Oft aber schienen die Krankheitsflüsse nach unten in den Thorax zu gehen, wo sie nach Weyers Ansicht eine nicht minder tödliche Pleuritis auslösten. Und manchmal gelangte der krankmachende Stoff (immer nach Weyer) auch in die Lunge. Vom Thorax aus wandte er sich oft zur Wirbelsäule, wo er starke Schmerzen auslöste, und bei den Frauen zum Uterus, wo die an sich schon durch den Monatsfluß an Blutung gewohnten Venen durchbrachen. Blutungen der Uterusschleimhaut sind übrigens eine auch uns bekannte Pestkomplikation.

Dies über den Verlauf der neuen Seuche, der pestilenzischen Angina, wie Weyer sie nannte. Im Vordergrund standen hochkontagiöse Anginen, die sich in eine Lungenseuche mit hauptsächlich pleuritischen Symptomen umwandeln konnte.

Nach einem bitterkalten Winter trat 1565 in Niederdeutschland wiederum eine unbekannte, tödliche Seuche auf, die Weyer als pestilenzische Pleuritis bezeichnete. Die meisten von ihr Befallenen starben wiederum in kurzer Zeit. Sie litten unter starker Atemnot, Seitenstechen und Auswurf, der oft blutig war. Als weitere Komplikationen traten Delirien auf.

Wenn wir bei den von Weyer unter dem Jahre 1564 beschriebenen «Anginen» und «Pleuritiden» noch zögern, die retrospektive Diagnose Pest zu stellen, so fällt uns dies viel leichter bei der unter dem Jahre 1565 beschriebenen Seuche. Vor allem, wenn wir Weyers Überlegungen zur Geschichte dieser Krankheit lesen: 
«Ich will nun auch die Geschichte dieser ansteckenden Krankheit in Erfahrung bringen, da sie bei keinem der alten Autoren beschrieben ist, ebensowenig, soviel ich weiß, von den neueren. Mit der einzigen Ausnahme jenes Guido von Chauliac, der der Nachkommenschaft als ewige Erinnerung (in cap.5, doct.2, tract. 2 seiner Chirurgia) eine ähnliche epidemische Seuche aufführt, welche 1348 ein überaus großes Unheil in Form eines Massensterbens für die ganze Welt brachte: Teils mit Kontinua-Fieber und Blutspucken, teils mit Anthrazes entweder in den Achseln oder in der Leiste. Durch die Fürsichtigkeit des erwähnten Autors kam später Valescus von Taranta ${ }^{6}$ in dunkle Kenntnis dieser seltenen Krankheit, wie der beigefügte Nachtrag seiner Abhandlung über die Pleuritis beweist.»

Eine ähnliche Seuche ereignete sich 1564 in Zürich: Unter dem 15. Februar schreibt Konrad Geßner an Theodor Zwinger in Basel, daß die Pest [wie in Basel] auch in Zürich nachgelassen habe ${ }^{7}$. An Johannes Krato von Krafftheim in Wien unter dem 5. März: «In Basel breitet sich die Pest etwas aus. Bei uns sterben viele an Pleuritis und Angina.» ${ }^{8}$ Am 24. Juni konnte Geßner seinem Freund Achilles Pirmin Gasser in Augsburg das Nachlassen der Seuche melden, welcher auch der ältere Froschauer erlegen war: «Für das, was Du mir über die Behandlung der Pleuritis geschrieben hast, sehr gelehrter Gasser, danke ich Dir. Diese Krankheit hat bei uns, Gott sei Dank, nachgelassen. Es war aber nicht die gewöhnliche, sondern jene ansteckende, pestartige und blutige Pleuritis.» ${ }^{9}$ Im Auftrag von Konrad Geßner schrieb Thaddeo Duno (1523-1613) eine Abhandlung über diese Pleuritis ${ }^{11}$ :

\section{"Wie die Beschaffenheit dieser Pleuritis beurteilt wurde.}

Wir meinen, daß diese Krankheit wirklich Pleuritis genannt werden muß, da ja entweder alle oder doch die meisten, wenigstens aber die unzertrennlich zur Pleuritis gehörenden Zeichen vorhanden waren. Ich nenne: Fieber; Seitenschmerz, ähnlich dem stechenden - obschon manchmal nicht so sehr akut (wir würden nämlich sagen, daß mehr die Muskeln als die aufliegende Membran befallen waren); erschwerte Atmung und bei manchen leicht blutiges Sputum und ein etwas harter Puls. Alles Zeichen, welche mit Sicherheit bei andern, nicht so verderblichen Pleuritiden aufzutreten pflegen und das pleuritische Syndrom darstellen. Da sie wirklich sehr viele angriff und sehr viele Symptome mit sich brachte, die Kräfte schnell niederwarf und rasch abtötete, wurde sie als bösartig und pestilenzisch betrachtet und als Sprößling der Pest, der sie wahrlich nicht unähnlich war. Weil viele in derselben Familie daran zugrunde gingen, stand auch klar fest, daß sie kontagiös war.» ${ }^{12}$ 
«... Insgesamt haben wir sie dennoch eher als fremdartige (notham), denn als bekannte (exquisitam) Pleuritis beurteilt, welche mehr die Muskeln als die Membran befällt und die vor allem durch schleimige Kochung, welcher Galle beigemischt ist, entstand. Beides machte sie bösartig und virulent. Weshalb, wenn jemand diese Krankheit nicht «pleuritis pestilens», sondern «pestis pleuritica» nennen möchte - gleich wie die andere Krankheit eher anginöse Pest und nicht pestilenzische Angina -, wir sagen würden: über Namen ist nicht zu streiten, wenn nur über die Tatsachen Einigkeit besteht. Wir sagen dies deshalb, weil die Pest in dem Teil des Körpers, wo sie sich am meisten entfacht hat, Entzündungen, Bubonen, Anthrazes und andere Schwellungen hervorbringt.» ${ }^{13}$

Als wie gefährlich die Ärzte die Krankheit einschätzten, zeigt der Abschnitt über die Therapie:

«Zum ersten kamen wir überein (wir, alle Zürcher Ärzte - d.h. Konrad Geßner natürlich, Georg Zeller, Kaspar Wolf und ich - waren nämlich auf Befehl des Magistrats zusammengekommen, um uns zu beraten, was gegen diese Art von Krankheit zu tun sei) - wir kamen überein, nicht zu den an dieser Krankheit Leidenden zu gehen, wenn wir nicht am ersten oder spätestens am zweiten Krankheitstag gerufen würden. Es sei denn, um einer andern, nichtärztlichen Aufgabe Willen. Nachdem nämlich der günstige Behandlungsmoment, welcher sich zu Beginn anbot, verstrichen war, hätten wir nur zu unserm - und bedenke! - auch zum schlechten Ruf der Kunst, unter höchster Gefahr und ohne Erleichterung für den Kranken, eine Behandlung beginnen können. Und dies vornehmlich bei unsern Leuten, die sehr wenig enthaltsam und auch sehr wenig gehorsam sind.» ${ }^{14}$

Ein so bemerkenswerter Beschluß war von den protestantischen Ärzten Zürichs während einer Bubonenpest nie gefaßt worden.

Dunus war demnach eher gegen die Bezeichnung Pestpleuritis, da die Beulen und Anthrazes fehlten. Aus demselben Grund stellte auch der Medizinhistoriker Meyer-Ahrens nicht die Diagnose Pest. Er hatte aber noch einen weiteren Grund: Mit seiner historischen Arbeit «Der Stich in den Jahren 1564 und 1565 in Zusammenhange mit den übrigen Epidemieen der Jahre 1562-1566» wollte er getreu der Krankheitssystematik von Johann Lukas Schönlein dartun, wie bei einer bestehenden «typhösen Konstitu- 
tion» aus katarrhalischen Vorläufern sich ein «Brusttyphus», später ein «Pesttyphus» (mit vorzugsweiser Beteiligung der Brustorgane) und in letzter Form, wie er meint, eine wahre Drüsenpest entwickeln kann ${ }^{15}$.

Wenn uns heute der Zusammenhang der verschiedenen damals auftretenden Epidemien sonst nicht mehr ganz einleuchtet, so sind wir um so dankbarer, daß Meyer-Ahrens mit höchster Akribie diese alle zusammengetragen hat. Denn so sehen wir, daß Bubonen- und Lungenpest oft zeitlich und örtlich abwechseln: im Spätsommer und Herbst die Bubonenpest, im Frühjahr die Lungenpest. Dies erklärt auch ein bislang nicht deutbares Phänomen: In sehr vielen Pestepidemiejahren nimmt die Sterblichkeit schon im Frühjahr zu, um dann wieder abzunehmen, bis im September die «Pest», wie in den Totenbüchern steht, zu wüten beginnt. Im Frühjahr finden wir dagegen nicht selten den Vermerk: «Lungensucht» oder «Bräune».

\section{Anmerkungen}

${ }^{1}$ Meyer-Ahrens, Konrad, Der Stich in den Jahren 1564 und 1565 im Zusammenhange mit den übrigen Epidemieen der Jahre 1562-1566. Zürich 1848.

${ }^{2}$ Weyer, Johannes, Medicarum Observationum Rararum Liber I. Zit. nach der Ausgabe: Apud Petrum Montanum, Amstelodami, Anno 1657. S.53-61. 1. Ausgabe: Oporinus, Basel 1567.

${ }^{3}$ Pollitzer, Robert, Review of recent literature on plague. Bulletin of the WHO. Vol.23, No.2-3 1960, S. 347.

${ }^{4}$ Guy de Chauliac, La Grande Chirurgie. Ed. E. Nicaise, Paris 1890. S. 167-177. Im Kommentar von Nicaise werden weitere zeitgenössische Autoren genannt.

${ }^{5}$ Weyer, op.cit. De variis Pestilentium defluxionum generibus, quae Anno 1564 \& 1565 apparuere in quibusdam Germaniae inferioris locis. S. 53-56.

${ }^{6}$ Valescus de Taranta kam um 1380 nach Montpellier. 1418 gab er das «Philonium pharmaceuticum et chirurgicum de medendis omnibus cum internis tum externis corporis affectionibus» heraus mit einem Anhang über die Behandlung der Pleuritis.

${ }^{7}$ Geßner, Konrad, Epistolae Medicinales, Zürich 1577, fol. 109v - 110 r.

8 ibid. fol. $17 \mathrm{v}$.

9 Peine, Hans, Briefe Konrad Geßners an seine Freunde Gasser und Culmann, Düsseldorf 1939, S. 35 .

${ }^{10}$ Geßner, op. cit. fol.21 r., Brief an Johannes Krato von Krafftheim, dat. 24. 7.1564.

${ }^{11}$ Dunus, Thadaeus, Epistolae Medicinales ... Eiusdem de Hemitritaeo ... Item, Miscellanorum de Re Medica liber ... alias non editus. Zürich 1592, fol. 130r-138r.

12 ibid. folg. $132 \mathrm{r}$.

13 ibid. fol. $132 \mathrm{v}-133 \mathrm{r}$.

14 ibid. fol. $133 \mathrm{r}-\mathrm{v}$.

15 Meyer-Ahrens, op. cit. «Rückblick», S. 176-182. 


\section{Summary}

An epidemic pulmonary disease raged in Europe 1564 and 1565. Konrad Meyer-Ahrens, a medical historian saw a connexion between pulmonary disease («Stich») and the bubonic plague of that time and classified those diseases as a species of thyphoid fevers (1848).

Johannes Weier as a contemporary physician remembered the description of Black Death by Guy de Chauliac 1348, when plague appeared in both forms, as pulmonary and as bubonic plague. Johannes Weier was convinced, that the "pleuritis pestilentialis" of 1564/65 is similar to the pulmonary plague of Guy de Chauliac. In the Swiss Confederation the disease was called "pleuritis pestilens" or "pestis pleuritica". Thus, the plague in the Europe of 16th century may have appeared in both, the bubonic and the pulmonary form.

Dr. med. Vera Waldis

Minervastraße 115

C.H-8032 Zürich 